\title{
A DECISION SUPPORT SYSTEM FOR THE MANAGEMENT OF COASTAL LAGOONS ${ }^{1}$
}

\author{
Marco Casini, Chiara Mocenni, Simone Paoletti, \\ Antonio Vicino
}

\author{
Dipartimento di Ingegneria dell'Informazione, \\ Università di Siena \\ Via Roma 56, 53100 Siena, Italy \\ \{casini,mocenni,paoletti,vicino\}@dii.unisi.it
}

\begin{abstract}
The correct management of coastal lagoon areas with anthropic exploitation is a difficult task for local authorities. Socio-economic interests and environment preservation are typically contrasting objectives between which a suitable trade-off must be achieved. The solution of this problem requires the development of interdisciplinary and multicriteria approaches. Motivated by future application to the Lagoon of Sacca di Goro (Italy), this paper proposes the architecture of a Decision Support System (DSS) for the management of coastal lagoon areas characterized by shellfish farming and agriculture. The DSS integrates a dynamic model for scenario simulations, the computation of several indicators for sustainability analysis and an optimization model for optimal resource allocation. Special emphasis is devoted to the definition of the optimization problem, which takes into account both the socio-economic and the environmental aspects. A numerical example shows the features of the proposed DSS. Copyright (c) 2005 IFAC
\end{abstract}

Keywords: Environmental system; Lagoon management; Optimization problem; Decision support system

\section{INTRODUCTION}

The development of management tools for coastal lagoons is a complex task requiring interdisciplinary research and active interaction with the end-users. Mathematical models of the biological, physical and chemical processes are fundamental tools for analyzing disruptions in the ecosystem due to abnormal conditions. Many efforts have been devoted, for instance, to simulation and prediction of eutrophic and dystrophic conditions leading to summer anoxic crises (see, e.g., Garulli et al. 2003, Zaldivar et al. 2003, and references therein). However, the successful management of

1 This research has been partially supported by the EU funded project DITTY in the Energy, Environment and Sustainable Development programme of the European Commission (EVK3-CT-2002-00084). such complex systems requires the integration of the information provided by mathematical models with other kinds of analysis. The socio-economic analysis assumes for instance great importance in coastal lagoons, especially where various kinds of anthropic pressures (aquaculture, fishery, tourism, etc.) are sources of conflicts among different users. Decision Support Systems (DSSs), i.e., information systems that help decision making, represent a suitable framework for the implementation of multicriteria approaches. They have been already successfully applied to the management of coastal basins (see, e.g., Pastres et al. 2001).

This paper proposes the structure of a DSS for the management of coastal lagoon areas characterized by shellfish farming and agriculture. These activities are responsible for important ecosystem disruptions, such as eutrophic conditions, algal 
blooms, oxygen depletion and nutrients production. The contrasting objectives of preserving the lagoon ecosystem, still guaranteeing the economic growth of the area, motivate the need for general management tools that take simultaneously into account both socio-economic and environmental aspects. The objective of the DSS is to provide local authorities with effective decision tools for quantifying and allocating the available resources (namely, the areas dedicated to agriculture and aquaculture) among the various producers. To this aim, the DSS exploits a dynamic model of the specific site for simulation of different scenarios, where a scenario is defined by the quantities of resources to be allocated. Sustainability analysis is then carried out by computing both ecological and socio-economic indicators. The solution of a suitable optimization problem provides the optimal resource allocation for each sustainable scenario. Finally, the best scenario is selected among all (optimized) sustainable scenarios according to a user-specified criterion.

The DSS proposed in this paper has been developed in view of its application to the management of the Lagoon of Sacca di Goro (Northern Adriatic Sea, Italy). This lagoon is one of the most important aquaculture areas in Italy, with about $10 \mathrm{~km}^{2}$ of aquatic surface (about $40 \%$ of the total) exploited for clam farming (Tapes philippinarum). The annual production of clams is about 15000 tons, for a revenue of 50 million Euros. Surrounding areas are heavily exploited for agriculture. These strong farming activities determine high nutrients loading, eutrophication, growth of seaweeds (especially macro-algae), and oxygen depletion. A biogeochemical model of the Lagoon of Sacca di Goro has been proposed by Zaldìvar et al. (2003).

The paper is organized as follows. Section 2 describes the overall structure of the DSS. Sections 3 and 4 focus on the sustainability analysis and the optimization model, respectively. A numerical example is illustrated in Section 5, and conclusions are drawn in Section 6 .

\section{DESCRIPTION OF THE DSS}

In a decision task, a scenario is defined by value assignments of all the parameters (key variables or options) that are involved in decision making. In the proposed DSS, the options are represented by the areas dedicated to agriculture and aquaculture. Such a choice is induced by the requests for new farming concessions that the local authorities are asked to grant. The farming areas are thus the resources that the local authorities (in the following, the decision maker) allocate among the producers. The scheme of the DSS is shown in Figure 1. It consists of four steps:

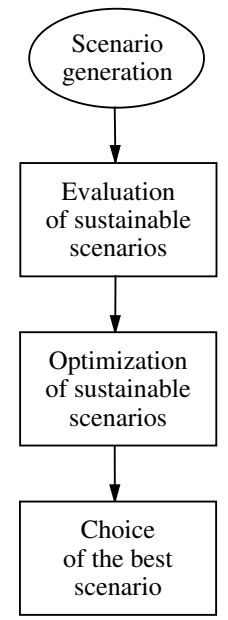

Figure 1. Architecture of the DSS for the management of a coastal lagoon.

1. Scenario generation. A set of candidate scenarios is generated by hypothesizing a number $n_{s}$ of different value assignments for the areas dedicated to agriculture and aquaculture.

2. Evaluation of sustainable scenarios. All scenarios are evaluated according to both socioeconomic and environmental criteria. If a given scenario does not satisfy one or more criteria, then it is rejected. Otherwise, it is accepted as sustainable.

3. Optimization of sustainable scenarios. For each sustainable scenario, the optimal allocation of the related resources among the producers is determined via the solution of a suitable linear program.

4. Choice of the best scenario. Among all the sustainable scenarios, the best scenario is selected according to the criterion that the decision maker specifies on the basis of economic, social and ecological considerations.

In the following sections, steps 2,3 and 4 will be described in detail.

\section{SUSTAINABILITY ANALYSIS}

The aim of the sustainability analysis is to evaluate whether a given scenario is satisfactory from both the socio-economic and the ecological point of view. To this purpose, it is helpful to consider a set of suitable indicators that simultaneously take into account both aspects. In this paper the sustainability analysis is carried out via the algorithm proposed in Figure 2, which mainly consists of three interconnected blocks:

- Site model. A dynamic model of the specific site is used to simulate the dynamics of the biological indicators (e.g., nutrients, macroalgae and oxygen), and other variables. 
- Environmental analysis. It provides ecological indicators to evaluate the health status of the environment.

- Socio-economic analysis. Suitable indicators are computed to evaluate costs and benefits of the considered scenario.

All the biological, ecological and socio-economic indicators are then compared with suitable thresholds, previously defined on the basis of regulations and experience. If a given scenario satisfies all the thresholds, then it is accepted as sustainable. Otherwise, it is rejected. The remainder of this section focuses on the sustainability analysis proposed for the Lagoon of Sacca di Goro, and addresses the description of the related variables and indicators. However, the scheme shown in Figure 2 is quite general, and can be adapted to any other site by specifying each block and the corresponding inputs and outputs.

\subsection{Description of the variables}

In the following, the subscript $i=1, \ldots, n_{s}$ indicates that the variables are related to the $i$-th scenario. The vector $V_{i}$ contains the parameters defining the $i$-th scenario. As described in Section 2 , it is composed by two values, namely the areas dedicated to agriculture and aquaculture. This vector is the control input to the dynamic model, whereas the vector $D$ represents exogenous inputs (e.g., temperature, light intensity, rainfall, wind speed, water inflow, etc.) to both the dynamic model and the environmental analysis. The output of the dynamic model is the vector $S_{i}$, monitoring the production and the physical, chemical and biological status of the lagoon ecosystem along a prediction horizon $T$. In particular, it contains the quantities of nutrients, macroalgae, phytoplankton, zooplankton and oxygen, as well as the shellfish production.

The vector $F_{i}$ represents other exogenous inputs to the environmental analysis, such as the fish population and the quantities of fuel and fertilizers consumed by economic activities. The vector $E_{i}$ contains the production values of all the economic sectors of interest, such as agriculture, fishery, aquaculture and food transformation. These are inputs to the socio-economic analysis, together with other inputs $G_{i}$ from the environmental analysis, e.g., the output exergy per economic sector and total (see Section 3.3). Note that some entries of $E_{i}$ and $F_{i}$ can be functions of $V_{i}$ and $S_{i}(e . g$., the production value of aquaculture is related to shellfish production).

The vector $I e_{i}$ is composed of the ecological indicators, e.g., the exergetic efficiency per economic sector and the total exergy (see again Section 3.3). The vector $I s_{i}$ contains the socio-economic indicators, e.g., the ratio of the output exergy to the production value per economic sector and total (see

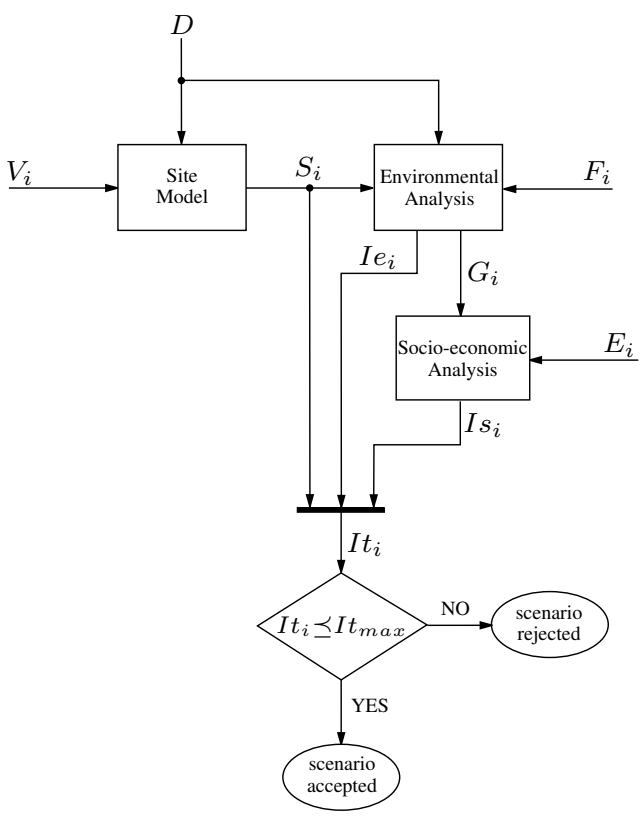

Figure 2. Block diagram of the algorithm for the sustainability analysis.

Section 3.4). The indicators $S_{i}, I e_{i}$ and $I s_{i}$ are finally combined into the vector $I t_{i}$ representing the global indicators. The vector $I t_{\max }$ contains the acceptance thresholds for $I t_{i}$.

\subsection{Site model}

For the Lagoon of Sacca di Goro, the biogeochemical model proposed in (Zaldivar et al., 2003) is used. It considers the nutrient cycles in the water column as well as in the sediments. Phytoplankton, zooplankton and macro-algae dynamics, as well as shellfish farming, are modelled. The dynamics of oxygen is also simulated in order to predict anoxic crises in the lagoon. Input fluxes from the watershed are considered, as well as water exchanges with the sea. Nutrients from the watershed, wet and dry deposition, temperature, light intensity and wind speed are considered as exogenous inputs.

Future work will concern the development of a simplified model for the lagoon ecosystem, like in (Allegretto et al., 2003). The aim is to build a model that is still able to capture the dynamics of interest, but is less sensitive to parameter variations and disturbances, and can be directly incorporated into the DSS. The most suitable model class to this purpose is under investigation.

\subsection{Environmental analysis}

The proposed environmental analysis for the Lagoon of Sacca di Goro is based on two different methodologies whose aim is to evaluate the modifications of the lagoon ecosystem induced by anthropic exploitation. These methodologies rely on the concepts of embodied exergy and exergetic efficiency. 
Embodied exergy. The thermodynamic definition of exergy is the amount of work that a system can perform by being brought into equilibrium with its environment. Exergy attempts to account for the actual free energy of the biomass by including the free energy that is stored in the information embodied in the biomass structure (genes). This makes it possible to use the exergy as a goal function (Bendoricchio and Jørgensen, 1997), as it measures the distance of an ecosystem from the equilibrium, i.e., from death. For the $i$-th scenario, the embodied exergy $E x_{t o t, i}$ of the ecosystem is computed as follows:

$$
E x_{t o t, i}=\sum_{h} \beta_{h} X_{h, i}
$$

where:

- $X_{h, i}$ is the biomass concentration of the $h$ th constituent (e.g., flora, fauna, organic and inorganic substances).

- $\beta_{h}$ is the specific exergy of the $h$-th constituent.

According to (1), the higher is the biodiversity, the larger is the value of $E x_{t o t, i}$, and hence the better is the health status of the ecosystem.

Exergetic efficiency. The exergetic efficiency is a measure of the consumption of renewable and non-renewable exergy related to the production of a given economic sector, where production is intended as the aggregate of marketable products (e.g., clams and mussels for aquaculture). For the $k$-th economic sector considered in the $i$-th scenario, the exergetic efficiency $\eta_{i}^{k}$ is computed as the ratio of the output exergy to the input exergy of the production process, i.e.:

$$
\eta_{i}^{k}=\frac{\sum_{p} \pi_{p, i}^{k} b_{p}^{\pi}}{\sum_{f} \phi_{f, i}^{k} b_{f}^{\phi}+\sum_{r} \rho_{r}^{k} b_{r}^{\rho}},
$$

where:

- $\pi_{p, i}^{k}$ is the quantity of the $p$-th product.

- $\phi_{f, i}^{k}$ is the flow of the $f$-th non-renewable input supporting the production process.

- $\rho_{r}^{k}$ is the flow of the $r$-th renewable input supporting the production process.

- $b_{p}^{\pi}, b_{f}^{\phi}, b_{r}^{\rho}$ are, respectively, the specific exergies of products, non-renewable inputs and renewable inputs.

The product quantities $\pi_{p, i}^{k}$ and the flows of nonrenewable inputs $\phi_{f, i}^{k}$ are provided by $F_{i}$, and partially by $S_{i}$. The flows of renewable inputs $\rho_{r}^{k}$ are provided by $D$.

\subsection{Socio-economic analysis}

Following Verdesca et al. (2003), the socio-economic analysis for the Lagoon of Sacca di Goro is carried out by computing, for each economic

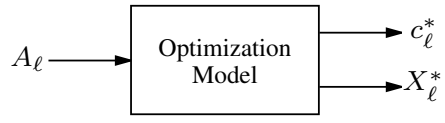

(a)

\begin{tabular}{|c|c|c|}
\hline $\begin{array}{c}\text { Scenario } \\
\text { Data }\end{array}$ & $\begin{array}{c}\text { Optimal } \\
\text { objective }\end{array}$ & $\begin{array}{c}\text { Optimal resource } \\
\text { allocation }\end{array}$ \\
\hline$A_{1}$ & $c_{1}^{*}$ & $X_{1}^{*}$ \\
\hline$A_{2}$ & $c_{2}^{*}$ & $X_{2}^{*}$ \\
\hline$\cdots$ & $\cdots$ & $\cdots$ \\
\hline$A_{N_{s}}$ & $c_{N_{s}}^{*}$ & $X_{N_{s}}^{*}$ \\
\hline
\end{tabular}

(b)

Figure 3. (a) Input/output scheme of the optimization model. (b) Table of sustainable scenarios $\left(N_{s} \leq n_{s}\right)$.

sector and for the whole ecosystem, the ratio of the Output Exergy to the Production Value (denoted by $O E / P V)$. This value can be interpreted as the amount of "health" returned to the system per unit of production value. Hence, the larger are the $O E / P V$ values, the more sustainable are the production activities for the environment.

\section{OPTIMIZATION MODEL}

Each sustainable scenario is characterized by a set of resources (in particular, the areas dedicated to agriculture and aquaculture) that the decision maker (the local authority) must allocate among the various actors (producers). To this aim, linear programming represents a simple but still powerful and flexible tool that allows to implement a wide range of objectives and constraints. The objective of the decision maker could be, for instance, the minimization of the pollution (cost function) subject to constraints (lower bounds) on the production levels, or vice versa the maximization of the production level (profit function) subject to constraints (upper bounds) on the pollutants. Additional constraints must be considered in order to guarantee that the solutions of the optimization problem are consistent with the supposed scenario.

Figure 3(a) shows the input/output scheme of the optimization model. For the $\ell$-th sustainable scenario, $\ell=1, \ldots, N_{s}$, it takes as inputs the scenario parameters, the exogenous inputs, and the sustainability indicators (vector $A_{\ell}$ ). These data are used to compute the coefficients and the bounds for the optimization problem, whose outputs are the optimal value $c_{\ell}^{*}$ of the objective function, and the vector $X_{\ell}^{*}$ containing the optimal allocation of the resources. The formulation of the optimization problem depends on the objective and the constraints specified by the decision maker. Section 5.2 illustrates a simple linear program for maximizing the production value subject 
Table 1. Areas dedicated to agriculture and aquaculture

\begin{tabular}{ccc} 
& $\begin{array}{c}\text { Agriculture } \\
{\left[\mathrm{m}^{2} \times 10^{8}\right]}\end{array}$ & $\begin{array}{c}\text { Aquaculture } \\
{\left[\mathrm{m}^{2} \times 10^{7}\right]}\end{array}$ \\
\hline $\mathcal{S}_{1}$ & 3.60 & 3.00 \\
$\mathcal{S}_{2}$ & 4.10 & 2.90 \\
$\mathcal{S}_{3}$ & 5.40 & 3.90 \\
$\mathcal{S}_{4}$ & 1.80 & 2.10 \\
\hline
\end{tabular}

Table 2. Exergetic efficiency per economic sector and total exergy

\begin{tabular}{cccc}
$\begin{array}{c}\text { Agriculture } \\
\times 10^{-3}\end{array}$ & $\begin{array}{c}\text { Aquaculture } \\
\times 10^{-5}\end{array}$ & $\begin{array}{c}\text { Total exergy } \\
{\left[\mathrm{J} \times 10^{15}\right]}\end{array}$ \\
\hline $\mathcal{S}_{1}$ & 1.73 & 7.64 & 3.28 \\
$\mathcal{S}_{2}$ & 1.62 & 7.69 & 2.83 \\
$\mathcal{S}_{3}$ & 1.50 & 6.55 & 2.05 \\
$\mathcal{S}_{4}$ & 1.81 & 7.75 & 3.25 \\
\hline
\end{tabular}

to constraints on social impact, pollutants, exergy and $O E / P V$ values. Following this example, more complex problems can be easily constructed.

\subsection{Choice of the best scenario}

Figure 3(b) shows a table containing the scenario data, the optimal value of the objective function and the optimal resource allocation for each sustainable scenario. This table can be used to carry out the choice of the best scenario. For instance, if the objective is to maximize the production value, the best scenario is selected by taking the maximum over the second column. Other criteria are however applicable, see, e.g., Section 5.3.

\section{NUMERICAL EXAMPLE}

In this section, a numerical example illustrates the implementation of the proposed DSS. The solutions obtained are analyzed and discussed. Four scenarios are considered, defined by the parameters shown in Table 1.

\subsection{Evaluation of sustainable scenarios}

The sustainability analysis is carried out for each scenario as described in Section 3. The values of the ecological indicators (exergetic efficiencies and total exergy) are reported in Table $2 . \mathcal{S}_{3}$ and $\mathcal{S}_{4}$ appear to be, respectively, the worst and the best scenario from the ecological point of view. The scenario $\mathcal{S}_{3}$ is rejected in this phase, since the good economic performances are not accompanied by equally good ecological performances. In particular, the increase of the farming areas determines higher nutrients concentrations, with a consequent appreciable decrease of the exergetic efficiency of aquaculture. However, in the following section the optimization is carried out also on this scenario in order to further highlight its infeasibility.

\subsection{Optimization of sustainable scenarios}

Five producers $\mathcal{P}_{j}, j=1, \ldots, 5$, in the aquaculture economic sector are considered. Their profiles are summarized in Table 3. Each producer profile is characterized by the production value $(P V)$, the social impact $(S I)$, and the output of nutrients $(N)$ and exergy $(O E)$. The social impact takes into account, e.g., the number of employees and the level of organization, and is given as a score between 1 and 10, where lower scores correspond to better social impact. The resource to be allocated among the producers is the area dedicated to aquaculture, and every decision variable $x_{j}$ of the optimization problem represents the fraction of the resource assigned to the $j$-th producer, $j=1, \ldots, 5$. For a given scenario, the objective is to maximize the production value subject to constraints on social impact, nutrients, exergy, and the ratio $O E / P V$. These constraints take the form of upper bounds on $S I$ and $N$, and lower bounds on $O E$ and $O E / P V$ (see Table 4). The considered optimization problem can be formulated as a linear program:

$$
\begin{aligned}
& \max \sum_{j=1}^{5} c_{j} x_{j} \quad(P V) \\
& \text { subject to } \\
& \sum_{j=1}^{5} a_{1, j} x_{j} \leq b_{1}^{(i)}(S I) \\
& \sum_{j=1}^{5} a_{2, j} x_{j} \leq b_{2}^{(i)}(N) \\
& \sum_{j=1}^{5} a_{3, j} x_{j} \geq b_{3}^{(i)}(O E) \\
& \sum_{j=1}^{5} a_{4, j}^{(i)} x_{j} \leq 0 \quad(O E / P \\
& \sum_{j=1}^{5} x_{j} \quad=1 \\
& x_{j} \geq 0 \quad \forall j=1, \ldots, 5 .
\end{aligned}
$$

For the $j$-th producer, the coefficients $c_{j}, a_{1, j}$, $a_{2, j}$ and $a_{3, j}$ are provided by Table 3 . Table 4 reports the bounds $b_{1}^{(i)}, b_{2}^{(i)}, b_{3}^{(i)}$ and $b_{4}^{(i)}$. The bound on $N$ is the output of nutrients from aquaculture provided by the dynamic model. The bound on $O E$ is the value of the output exergy from aquaculture provided by the environmental analysis. The bound on $O E / P V$ is the ratio of the output exergy to the production value for the aquaculture economic sector; this is provided by the socio-economic analysis. All the bounds are given per unit of area. The coefficients $a_{4, j}^{(i)}$ of the $O E / P V$ constraint are computed as follows:

$$
\frac{\sum a_{3, j} x_{j}}{\sum c_{j} x_{j}} \geq b_{4}^{(i)} \Rightarrow \sum \overbrace{\left(b_{4}^{(i)} c_{j}-a_{3, j}\right)}^{a_{4, j}^{(i)}} x_{j} \leq 0 .
$$

The superscript $i$ denotes dependence on the scenario: $i=1, \ldots, 4$ in this example. 
Table 3. Producer profiles

\begin{tabular}{ccccc} 
& $\begin{array}{c}P V, c_{j} \\
{\left[€ / \mathrm{m}^{2}\right]}\end{array}$ & $S I, a_{1, j}$ & $\begin{array}{c}N, a_{2, j} \\
{\left[\mathrm{Kg} / \mathrm{m}^{2}\right]}\end{array}$ & $\begin{array}{c}O E, a_{3, j} \\
{\left[\mathrm{MJ} / \mathrm{m}^{2}\right]}\end{array}$ \\
\hline $\mathcal{P}_{1}$ & 0.30 & 2 & 8.85 & 0.50 \\
$\mathcal{P}_{2}$ & 0.12 & 8 & 20.60 & 0.32 \\
$\mathcal{P}_{3}$ & 0.35 & 6 & 16.43 & 0.41 \\
$\mathcal{P}_{4}$ & 0.20 & 6 & 12.50 & 0.47 \\
$\mathcal{P}_{5}$ & 0.18 & 3 & 14.80 & 0.45 \\
\hline
\end{tabular}

Table 4. Bounds of the optimization problem

\begin{tabular}{ccccc} 
& $S I, b_{1}^{(i)}$ & $\begin{array}{c}N, b_{2}^{(i)} \\
{\left[\mathrm{Kg} / \mathrm{m}^{2}\right]}\end{array}$ & $\begin{array}{c}O E, b_{3}^{(i)} \\
{\left[\mathrm{MJ} / \mathrm{m}^{2}\right]}\end{array}$ & $\begin{array}{c}O E / P V, b_{4}^{(i)} \\
{[\mathrm{MJ} / €]}\end{array}$ \\
\hline $\mathcal{S}_{1}$ & 4 & 13.35 & 0.426 & 2.29 \\
$\mathcal{S}_{2}$ & 4 & 12.11 & 0.428 & 1.85 \\
$\mathcal{S}_{3}$ & 4 & 7.52 & 0.364 & 1.96 \\
$\mathcal{S}_{4}$ & 4 & 17.73 & 0.428 & 2.31 \\
\hline
\end{tabular}

Table 5. Solutions of the optimization problem

\begin{tabular}{ccccccc} 
& $c^{*}$ & $x_{1}^{*}$ & $x_{2}^{*}$ & $x_{3}^{*}$ & $x_{4}^{*}$ & $x_{5}^{*}$ \\
\hline $\mathcal{S}_{1}$ & 0.203 & 0.125 & 0 & 0 & 0.375 & 0.5 \\
$\mathcal{S}_{2}$ & 0.267 & 0.667 & 0 & 0 & 0.333 & 0 \\
$\mathcal{S}_{3}$ & - & - & - & - & - & - \\
$\mathcal{S}_{4}$ & 0.199 & 0.103 & 0 & 0 & 0.367 & 0.530 \\
\hline
\end{tabular}

The solutions of the optimization problem (3) for each scenario are shown in Table 5, where $c^{*}$ denotes the production value at the optimum, and $x_{j}^{*}, j=1, \ldots, 5$, are the optimizers. Note that the instance of problem (3) corresponding to the scenario $\mathcal{S}_{3}$ is infeasible. This means that such a scenario is impracticable with the producer profiles at hand. Recall that $\mathcal{S}_{3}$ is also rejected according to the sustainability analysis, as it is the worst scenario from the ecological point of view. The producers $\mathcal{P}_{2}$ and $\mathcal{P}_{3}$, that are characterized by the worst profiles (bad social impact, high nutrients production, low output exergy), are never assigned with any available resource, although $\mathcal{P}_{3}$ would guarantee the best economic performance. Conversely, the producer $\mathcal{P}_{1}$, that has the best profile, is always assigned with sensible fractions of the available resources. The producers $\mathcal{P}_{4}$ and $\mathcal{P}_{5}$ have comparable average profiles, but the higher output of nutrients penalizes $\mathcal{P}_{5}$ with respect to $\mathcal{P}_{4}$ in the scenario $\mathcal{S}_{2}$. It is interesting to note that the $O E / P V$ constraint is active at the optimum of all the considered optimization problems, and hence it is effective in achieving the desired trade-off between economic and ecological objectives by avoiding solutions were the production maximization is dominant.

\subsection{Choice of the best scenario}

In this example the best scenario is finally selected by taking the maximum over the first column of Table 5, since production maximization under environmental constraints is the objective of the decision maker. According to this criterion, the scenario $\mathcal{S}_{2}$ is the most preferable, because it guarantees the highest production value, still satisfying the ecological requirements. Other criteria could be also applied. For instance, the decision maker could prefer the scenario $\mathcal{S}_{1}$, that is characterized by a lower production value but divides the available resources among more producers. This could be desirable to avoid that few producers dominate the market.

\section{CONCLUSIONS}

In this paper, the structure of an integrated decision support system for the management of coastal lagoon areas has been presented. Motivated by the future application to the Lagoon of Sacca di Goro, the DSS takes into account both the ecological and the socio-economic aspects that are related to shellfish farming and agriculture. Its aim is to help local authorities in decision making concerning the grant of new farming concessions. This is achieved by integrating biological modelling, sustainability analysis and optimization techniques. Future work will concern the implementation and the validation of the DSS on field data from the Lagoon of Sacca di Goro. A simplified dynamic model, as well as additional sustainability indicators, will be also developed. The integration of the DSS with other kinds of multicriteria and scenario analysis will be further investigated.

\section{REFERENCES}

Allegretto, W., C. Mocenni and A. Vicino (2003). Periodic solutions and simulation for a lagoon model. In: Proc. Int. Workshop on Scientific Computation and Simulation. Hong Kong.

Bendoricchio, G. and S.E. Jørgensen (1997). Exergy as goal function of ecosystems dynamics. Ecological Modelling 102(1), 5-15.

Garulli, A., C. Mocenni, A. Tesi and A. Vicino (2003). Integrating identification and qualitative analysis for the dynamic model of a lagoon. International Journal of Bifurcation and Chaos 13(2), 357-374.

Pastres, R., C. Solidoro, G. Cossarini, D. Melaku Canu and C. Dejak (2001). Managing the rearing of Tapes philippinarum in the lagoon of Venice: A decision support system. Ecological Modelling 138(1-3), 231-245.

Verdesca, D., L. Torsello, M. Federici and R. Basosi (2003). Economic and exergy accounting for coastal lagoon systems: An integrated approach. In: Proc. Int. Conf. on Southern European Coastal Lagoons. Ferrara, Italy.

Zaldivar, J.M., E. Cattaneo, M. Plus, C.N. Murray, G. Giordani and P. Viaroli (2003). Long-term simulation of main biogeochemical events in a coastal lagoon: Sacca di Goro (Northern Adriatic Coast, Italy). Continental Shelf Research 23(17-19), 1847-1875. 